\title{
Grão de soja cru e inteiro na alimentação de bovinos: Excreção de grão de soja nas fezes
}

\author{
Rennó, F.P. ${ }^{1}$; Cônsolo, N.R.B. ${ }^{\prime}$; Barletta, R.V. ${ }^{1}$; Ventureli, B. ${ }^{2}$; Gardinal, R. ${ }^{\text {; }}$ Takiya, C.S. ${ }^{3}$; Gandra, J.R. ${ }^{3}$; e Pereira, A.S.C. ${ }^{1}$
}

'Departamento de Nutrição e Produção Animal VNP. FMVZ. USP. Pirassununga. SP. Brasil.

${ }^{2}$ Coordenação técnica de Ruminantes Poli-Nutri Nutrição Animal. Vila Menk. Osasco. SP. Brasil.

${ }^{3}$ Faculdade de Ciências Agrárias. Universidade Federal da Grande Dourados. MS. Brasil.

\section{PalaVRas ChaVe ADICIONAIS}

Ácidos graxos.

Digestibilidade.

Semente oleaginosa.

\section{ADDITIONAL KEYWORDS}

Fatty acids.

Digestibility.

Oilseed.

\section{INFORMACIÓN}

\section{Cronología del artíiculo.}

Recibido/Received: 15.1.2015

Aceptado/Accepted: 28.8.2015

On-line: 10.12 .2015

Correspondencia a los autores/Contact e-mail:

francisco.renno@usp.br

\section{RESUMO}

O objetivo desse estudo foi de avaliar os efeitos da inclusão de níveis crescentes de grão de soja cru inteiro (GS) em dietas de bovinos de corte e de vacas leiteiras de alta e média produção sobre o consumo de matéria seca (CMS), digestibilidade aparente total dos nutrientes, excreção e composição do grão de soja nas fezes. Para esse estudo foram realizados três ensaios em delineamento experimental de quadrado Latino $4 \times 4$, com 14 dias de adaptação e 4 dias de coleta de amostras. Os ensaios utilizaram as seguintes categorias animal: 1) 12 novilhos Nelore castrados $(420 \pm 28,5 \mathrm{~kg}$ de peso vivo, $24 \pm 3,5$ meses) agrupados em três quadrados latinos; 2) 12 vacas Holandesas $(580 \pm 23,5 \mathrm{~kg}$ de peso vivo, $90 \pm 14,6$ DEL e $30 \pm 2,7 \mathrm{~kg} / \mathrm{d}$ de produção de leite); e 3) 16 vacas Holandesas $(558 \pm 43,8 \mathrm{~kg}$ de peso vivo, $228 \pm 43,8 \mathrm{DEL}$, e $23 \pm 4,6 \mathrm{~kg} / \mathrm{d}$ de produção de leite). As dietas experimentais nos ensaios 1 e 2 apresentavam $0,8,16$ e $24 \%$ de inclusão de GS na dieta (com base na MS). O ensaio 3 apresentou dietas com inclusão de GS na MS da dieta de 0, 9, 18 e 27\% (G0, G9, G18 e G27, respectivamente). A inclusão de GS diminuiu $(p<0,05)$ o consumo de matéria seca das diferentes categorias animal. No entanto, as digestibilidades da MS e FDN não foram influenciadas $(p>0,05)$ pela inclusão de altos níveis de GS. O ganho médio diário dos novilhos Nelore e a produção de leite corrigida para $3,5 \%$ de gordura (PLC) das vacas não foram influenciados $(p>0,05)$ pela adição de GS nas dietas. $O$ consumo e a excreção de GS aumentaram linearmente $(p<0,01)$ nos trêss ensaios conforme a inclusão de GS na dietas. A composição bromatológica do GS excretado nas fezes não foi alterada $(p>0,05)$, independente da concentração de GS na dieta. De acordo com os estudos avaliados, níveis crescentes de grão de soja cru e inteiro podem ser utilizados na dieta de bovinos sem que ocorra mal aproveitamento nutricional do grão de soja e prejuízos a produção do animal.

\section{Whole raw soybean in cattle diet: Soybean output in feces}

\section{SUMMARY}

Our objective was to evaluate the effects of increasing doses of whole raw soybeans (WRS) in beef steer and dairy cow (high and medium milk yield) diets on dry matter intake (DMI), nutrient total apparent digestibility, excretion and composition of soybeans particles on feces. Three trials were performed into replicated $4 \times 4$ Latin square design, with $14 \mathrm{~d}$ of adaptation and $4 \mathrm{~d}$ of sampling. Trials were composed by the follow animal categories: 1) 12 Nellore steers, with average live weight of $420 \pm 28.5 \mathrm{~kg}$, and $24 \pm 3.5$ months age; 2) 12 Holstein cows, with average body weight of $580 \pm 23.5 \mathrm{~kg}, 90 \pm 14.6 \mathrm{DIM}$ and $30 \pm 2.7 \mathrm{~kg} / \mathrm{d}$ of milk yield; 3) 16 Holstein cows, with average body weight of $580 \pm \mathrm{kg}, 228 \pm \mathrm{DIM}$ and $23 \pm \mathrm{kg} / \mathrm{d}$ of milk yield;. The experimental diets in the trials 1 and 3 were: $\mathrm{G} 0$ ) diet without WRS; G8) inclusion of $8 \%$ of WRS on diet DM basis; G16) inclusion of $16 \%$ of WRS on diet DM basis; and G24) inclusion of $24 \%$ of WRS on diet DM basis. In the trial 2 were use diets containing $0,9,18$ and $27 \%$ of WRS inclusion on diet DM basis $(G 0, G 9, G 18$ and G27, respectively). The WRS inclusion decreased $(p<0.05)$ the DMI regardless of animal category. However, DM and NDF digestibility were not affected ( $p>0.05$ ) by increasing doses of WRS in diets. Average body weight gain of Nellore steers and fat corrected milk of Holstein cows were not altered $(p>0.05)$ by WRS diet inclusion. Whole raw soybeans intake and excretion of soybeans particles increased linearly $(p<0.01)$ according to WRS inclusion in all the trials. Chemical composition of soybeans particles excreted on feces was not altered ( $p>0.05$ ) by treatments, regardless of the dietary WRS. According to the studies, increasing levels of whole raw soybean may be added to cattle diets without decrease in nutrient utilization and impair animal performance. 


\section{INTRODUÇÃO}

A tecnificação e a intensificação do processo produtivo na pecuária de corte e leite são ferramentas utilizadas a fim de aumentar o potencial produtivo. A prática do confinamento vem sendo utilizada como alternativa na terminação de novilhos e para vacas de leite de alto potencial produtivo, no entanto apesar de vantajoso, o confinamento é uma atividade que pode encarecer o sistema de produção. De modo geral, em sistema de confinamento a alimentação é composta por comoddities, dentre eles destacam-se o fubá de milho e o farelo de soja, como fonte energética e proteica, respectivamente; fazendo com que a alimentação seja responsável por 70 a $80 \%$ dos custos de produção nesse sistema (Paulino et al., 2002). Por essa razão pesquisas que exploram o potencial de alimentos alternativos ou não processados, a fim de diminuir o custo das dietas e sem prejudicar a produção animal, tornam-se pertinentes.

Nesse contexto, destacam-se os grãos inteiros e sementes de oleaginosas, em que, o processamento dos mesmos (quebra, moagem, extrusão e peletização) encarecem o produto e consequentemente o custo de produção. Desta forma, o uso de grão inteiros vem tomando espaço na alimentação de ruminantes (Millen, 2009). As sementes de oleaginosas vêm sendo utilizadas como fonte proteica e energética pelo elevado teor de lipídio presente no interior da semente, o qual é envolto por uma matriz proteica que pode previnir uma rápida liberação do conteúdo lipídico da semente no ambiente ruminal e diminuir os efeitos negativos sobre a digestão ruminal da fibra. Além disso, o grão de soja cru e inteiro pode ser utilizado sem prejuízos a digestibilidade de nutrientes pois os microorganismos ruminais podem neutralizar a maior parte dos componentes anti-nutricionais, como exemplo a anti-tripsina (McDonald et al., 1995).

Dentre as diferentes sementes oleaginosas disponíveis no mundo, o grão de soja se destaca pela grande disponibilidade no território brasileiro. Quando se utiliza sementes inteiras na alimentação animal, frequentemente observa-se fragmentos da semente ou até mesmo sementes inteiras nas fezes dos animais, o que causa a impressão que o alimento não foi bem aproveitado pelo organismo, e que parte dos nutrientes contidos nas sementes foram perdidos nas fezes. No entanto, não existem estudos que descrevem a excreção e composição desse ingrediente presente nas fezes e que o correlacionam com a digestibilidade da dieta para fundamentar a ideia de que o ingrediente está sendo subutilizado.

Recentemente, Côrtes et al. (2015) avaliaram a composição das partículas de sementes de linhaça recuperadas das fezes de vacas recebendo dietas com níveis crescentes de semente de linhaça e não encontraram diferenças na composição bromatólogica dessas partículas.

O objetivo deste estudo foi de avaliar os efeitos da inclusão de níveis crescentes de grão de soja em dietas de bovinos de corte e de vacas leiteiras de alta e média produção sobre o CMS, digestibilidade dos nutrientes, excreção e composição das partículas do grão de soja nas fezes dos animais.

\section{MATERIAL E MÉTODOS}

\section{LOCAL DE EXECUÇÃO E ANIMAIS}

O estudo foi conduzido no Laboratório de Pesquisa em Bovinos de Leite da Faculdade de Medicina Veterinária e Zootecnia - Universidade de São Paulo, Pirassununga, Brasil. Esse estudo foi composto por três ensaios experimentais, como seguem: 1) foram utilizados 12 animais novilhos Nelore castrados $(420 \pm 28,5 \mathrm{~kg}$ de peso vivo, $24 \pm 3,5$ meses) agrupados em três quadrados latinos $4 \times 4$, contemporâneos e balanceados de acordo com o peso vivo. Os animais foram alimentados com quatro dietas durante o período experimental, formuladas para serem isoprotéicas de acordo com o sistema Cornell Net Carbohydrate and Protein System versão 5,$0 ; 2$ ) foram utilizadas 12 vacas da raça Holandesa (580 $\pm 23,5 \mathrm{~kg}$ de peso vivo, $90 \pm 14,6$ DEL e $30 \pm 2,7 \mathrm{~kg} / \mathrm{d}$ de produção de leite) agrupadas em três quadrados latinos $4 \times 4$, contemporâneos e balanceados de acordo com o período de lactação, produção de leite e peso vivo. Os animais foram alimentados com quatro dietas durante o período experimental, formuladas para serem isonitrogenadas, conforme recomendações do NRC (2001); and 3) foram utilizadas 16 vacas da raça Holandesa (558 $\pm 43,8 \mathrm{~kg}$ de peso vivo, $228 \pm 43,8 \mathrm{DEL}$, e $23 \pm 4,6 \mathrm{~kg} / \mathrm{d}$ de produção de leite) agrupadas em quatro quadrados latinos $4 \times 4$, contemporâneos e balanceados de acordo com o período de lactação, produção de leite e peso vivo. Os animais foram alimentados com quatro dietas durante o período experimental, formuladas para serem isonitrogenadas, conforme as recomendações do NRC (2001). Os três experimentos foram constituídos por quatro períodos, com duração de 18 dias cada um, sendo os 14 primeiros dias de adaptação às rações e os demais para coleta de amostras e dados.

\section{Dietas e manejo}

Nos três ensaios experimentais os animais foram alojados em galpão do tipo free-stall com ventilação forçada, baias individuais contendo camas de areia, bebedouros automáticos e cochos de alimentação. Os animais foram alimentados duas vezes ao dia, às 7:00 e 14:00 h. Nos experimento 1 e 2 as dietas possuiram a mesma inclusão do grão de soja: G0) Controle, composto por ração sem a inclusão de grão de soja; G8) inclusão de 8\%, G16) inclusão de 16\% e G24) inclusão de $24 \%$ do grão de soja cru e inteiro (com base na MS da dieta). As dietas do experimento 3 foram: G0) Controle, composto por ração sem a inclusão de grão de soja; G9) inclusão de 9\%, G18) inclusão de $18 \%$ e G27) inclusão de $27 \%$ do grão de soja cru e inteiro (com base na MS da dieta). A silagem de milho foi utilizada como fonte de volumoso nos 3 ensaios experimentais, sendo a proporção de volumoso:concentrado de 40:60 no experimento $1 ; 50: 50$ no experimento 2 e 60:40 no experimento 3. A composição bromatológica das dietas experimentais encontra-se nas tabelas I e II.

As vacas foram ordenhadas mecanicamente duas vezes ao dia às 06:30 e 15:30 h. A produção de leite foi registrada diariamente por medidor automático 
Tabela I. Composição bromatológica das dietas experimentais dos estudos 1 e 3 (confinamento de bovinos de corte e vacas de alta produção de leite) (Chemical composition of experimental treatments of trials 1 and 3 (feedlot and dairy cows of high milk yield)).

\begin{tabular}{|c|c|c|c|c|}
\hline \multirow[b]{2}{*}{ Item } & \multicolumn{4}{|c|}{ Dieta $^{1}$} \\
\hline & G0 & G8 & G16 & G24 \\
\hline \multicolumn{5}{|c|}{ Ensaio 1 - Confinamento novilhos de corte } \\
\hline Matéria seca ${ }^{1}$ & 64,01 & 64,42 & 64,85 & 65,13 \\
\hline Proteína bruta ${ }^{2}$ & 13,42 & 13,17 & 13,28 & 13,32 \\
\hline Eextrato eteéreo ${ }^{2}$ & 2,02 & 3,50 & 4,93 & 6,42 \\
\hline Fibra detergente neutro ${ }^{2}$ & 29,39 & 29,65 & 30,01 & 30,52 \\
\hline Carboidrato não-fibroso ${ }^{2}$ & 48,63 & 48,47 & 48,35 & 45,75 \\
\hline Energia líquida (Mcal/kg de MS) & 1,67 & 1,70 & 1,73 & 1,80 \\
\hline \multicolumn{5}{|c|}{ Ensaio 2 - Vacas de alta produção } \\
\hline Matéria seca ${ }^{1}$ & 62,01 & 62,17 & 62,22 & 62,03 \\
\hline Proteína bruta ${ }^{2}$ & 16,95 & 16,97 & 16,69 & 16,67 \\
\hline Extrato etéreo ${ }^{2}$ & 3,02 & 4,46 & 5,9 & 7,2 \\
\hline Fibra detergente neutro ${ }^{2}$ & 33,03 & 33,45 & 33,93 & 32,81 \\
\hline Carboidrato não-fibroso² & 42,14 & 40,33 & 38,99 & 38,74 \\
\hline $\begin{array}{l}\text { Energia líquida de lactação } \\
\text { (Mcal/kg de MS) }\end{array}$ & 1,72 & 1,75 & 1,79 & 1,84 \\
\hline
\end{tabular}

G0 (Controle); G8, G16 e G24 se referem a inclusão de, respectivamente, 8,16 e $24 \%$ de grão de soja cru integral na dieta (com base na MS). ${ }^{1} \%$ da matéria natural; ${ }^{2} \%$ da matéria seca.

Tabela II. Composição bromatológica das dietas experimentais do estudo 2 (vacas de média produção) (Chemical composition of experimental treatments of trial 2 (dairy cows of medium milk yield)).

\begin{tabular}{lrrrr}
\hline \multirow{2}{*}{ Item } & \multicolumn{4}{c}{ Dieta } \\
\cline { 2 - 5 } & \multicolumn{1}{c}{ G0 } & \multicolumn{1}{c}{ G9 } & G18 & G27 \\
\hline Matéria seca $^{1}$ & 52,9 & 53,18 & 53,4 & 53,8 \\
Proteína bruta $^{2}$ & 17,9 & 17,8 & 17,8 & 17,8 \\
Extrato etéreo $^{2}$ & 1,63 & 3,26 & 4,92 & 6,59 \\
Fibra detergente neutro $^{2}$ & 37,63 & 38,41 & 39,21 & 39,92 \\
Carboidrato não-fibroso $^{2}$ & 36,64 & 33,52 & 30,39 & 27,57 \\
Energia líquida de lactação & 1,72 & 1,80 & 1,88 & 1,96 \\
(Mcal/kg de MS) & & & &
\end{tabular}

G0 (Controle); G9, G18 e G27 se referem a inclusão de, respectivamente, 9,18 e $27 \%$ de grão de soja cru integral na dieta (com base na MS). ${ }^{1} \%$ da matéria natural. ${ }^{2} \%$ da matéria seca.

de leite (ICAR, DeLaval - Tumba, Suécia). As amostras de leite foram obtidas de maneira proporcional as duas ordenhas diárias durante o período de coleta. As amostras foram analisadas para o teor de gordura (Milkoscan, Foss Eltric, Hillerod - Denmark), e a PLC foi calculada de acordo com Sklan et al. (1992). Os novilhos foram pesados no início do experimento e ao fim de cada período experimental, em jejum sólido de 12 horas, a fim de se avaliar o ganho de peso médio diário (GMD).

\section{ANÁLISE DOS ALIMENTOS}

Diariamente, antes do fornecimento da dieta aos animais, foram feitas pesagens das sobras de cada animal a fim de estimar o consumo individual. Os animais foram alimentados de acordo com o consumo de matéria seca do dia anterior, de forma a ser mantido o percentual de sobras entre 5 e $10 \%$ do fornecido. Ao longo dos 4 dias do período de coleta, amostras de silagem, dos ingredientes do concentrado e sobras foram coletadas e acondicionadas em sacos plásticos e armazenadas a $-20^{\circ} \mathrm{C}$, para posterior análise bromatológica.

Os teores de matéria seca (MS, AOAC 950.15), extrato etéreo (EE, AOAC 920.39) e proteína bruta (PB, AOAC 984.13) nos alimentos e sobras foram analisados de acordo com as metodologias descritas por AOAC (2000). Os teores de fibra detergente neutro (FDN) foram obtidos conforme método descrito por Van Soest and Mason (1991), utilizando-se $\alpha$-amilase sem adição de sulfito de sódio em determinador de fibra (Analisador de Fibra TE-149, Tecnal - Equipamentos para Laboratório, Piracicaba - Brasil). Os teores de carboidratos não-fibrosos (CNF) foram estimados segundo Hall (1998), onde: $C N F=100-[(\% \mathrm{~PB}-\% \mathrm{~PB}$ da ureia + $\%$ Ureia) $]+\% \mathrm{EE}+\% \mathrm{MM}+\% \mathrm{FDN}$. A energia líquida foi calculada de acordo com o NRC (2001).

\section{EXCREÇ̃̃O DE FEZES E COEFICIENTE DE DIGESTIBILIDADE}

Para a determinação da composição das fezes, amostras $(0,5 \mathrm{~kg}$ com base na matéria úmida) foram coletadas diretamente do reto nos dias 15,16 e 17 de cada período experimental, antes da alimentação do período da manhã e da tarde. As amostras foram misturadas de modo a formar uma amostra composta $(1,5$ $\mathrm{kg}$ com base na matéria úmida) para cada animal por período e armazenadas a $-20^{\circ} \mathrm{C}$. A fibra indigestível em detergente ácido (FDAi) foi utilizada como marcador interno para estimar a excreção diária de fezes e para o cálculo do coeficiente de digestibilidade da MS e da FDN. Amostras de alimentos, sobras e fezes foram parcialmente secas em estufa com ventilação forçada a $55^{\circ} \mathrm{C}$ durante 72 horas e moídas em moinho de facas para passarem por uma peneira de $2 \mathrm{~mm}$ (Wiley mill, Arthur H. Thomas, Philadelphia, PA, USA). As amostras foram colocadas em sacos de tecido não tecido $\left(100 \mathrm{~g} / \mathrm{m}^{2}\right)$ de acordo com as recomendações de $20 \mathrm{mg}$ de amostra seca por $\mathrm{cm}^{2}$ (Nek, 1988) e incubadas no rumen de duas vacas secas, previamente adaptadas as dietas de cada ensaio experimental por $264 \mathrm{~h}$, de acordo com a metodologia descrita por Casali et al. (2008). Após 264 h os sacos foram lavados em água corrente, secos em estufa de ventilação forçada por 72 horas e submetidos ao tratamento de detergente ácido (Mertens, 2002). A excreção diária de fezes foi calculada como segue: Excreção fecal de MS $(\mathrm{kg} / \mathrm{d})=$ consume de FDAi/concentração de FDAi nas fezes. As amostras parcialmente secas de fezes também foram moídas em moinho de facas para passarem em peneira de $1 \mathrm{~mm}$ para posterior análise de MS e FDN como previamente descrito na seção anterior.

\section{EXCREÇÃO DE GRÃO DE SOJA NAS FEZES}

Amostras de fezes foram coletadas diretamente do reto nos dias 15, 16 e 17 de cada período experimental, antes da alimentação do período da manhã e da tarde, a fim de avaliar a excreção e composição do grão. Em seguida, foi realizada pesagem de aproximadamente 400 $\mathrm{g}$ de fezes em balança analítica. As amostras foram submetidas à lavagem em peneira de $4 \mathrm{~mm}$, as partículas 
de grão de soja foram coletadas manualmente, pesadas e armazenadas a $-20^{\circ} \mathrm{C}$, para posterior análise bromatológica. Foram analisados os teores de MS (AOAC 950.15), matéria orgânica (MO), matéria mineral (MM, 942.05), EE (AOAC, 920.39) e de PB (AOAC 984.13) nas partículas de grão de soja presentes nas fezes, de acordo com as metodologias descritas AOAC (2000). Os teores de FDN e CNF foram obtidos conforme previamente descrito na sessão de análise de alimentos.

O consumo de grão de soja com base na MS (kg/ dia) foi calculado pela fórmula: $\mathrm{CMS}_{\mathrm{gs}}=(\mathrm{CMS} \times \%$ grão de soja na dieta)/100. O consumo de matéria natural (CMN) de GS (kg/dia) foi obtido pela fórmula: $\mathrm{CMN}=$ (consumo de matéria seca de grão de soja)/MS de grão de soja. A concentração de grãos de soja excretados nas fezes (com base na MS) foi obtida pela equação: EMS= (consumo de MS do grão de soja - excreção de MS do grão de soja)/ excreção total de fezes*100, a excreção de grão de soja (com base na MN) foi obtida pela equação: (peso da amostra GS coletado*100)/peso amostra coletada de fezes MN. A excreção de MS (kg/dia) foi obtida pela equação: $\mathrm{EMS}=($ excreção total de fezes $\times \%$ grão de soja nas fezes)/100. A matéria natural corrigida foi obtida pela equação: $\mathrm{MNC}=($ excreção de $\mathrm{MS}(\mathrm{kg} /$ dia))/MS do grão de soja.

\section{ANÁLISE ESTATíSTICA}

Os dados obtidos foram submetidos ao SAS (Versão 9.1.3, SAS Institute, Cary, NC 2004), verificando a normalidade dos resíduos e a homogeneidade das variâncias pelo PROC UNIVARIATE.

Os dados foram analisados, pelo PROC MIXED de acordo com a seguinte modelo:

$$
Y_{i j k y}=\mu+Q_{i}+A_{j}+P_{y}+T_{k}+e_{i j y k}
$$

Onde:

$$
\mathrm{Y}_{\mathrm{ijyk}}=\text { variável dependente, }
$$

$\mu=$ média geral,

$\mathrm{Q}_{\mathrm{i}}=$ efeito de quadrado,

$\mathrm{A}_{\mathrm{j}}=$ efeito de animal,

$\mathrm{P}_{\mathrm{y}}=$ efeito do período,

$\mathrm{T}_{\mathrm{k}}=$ efeito do tratamento, $\mathrm{e}$

$\mathrm{e}_{\mathrm{ijk}}=$ efeito residual.

Efeito aleatório $A_{i}\left(Q_{i}\right)=$ animal dentro de quadrado. Os graus de liberdade foram calculados de acordo com o método satterthwaite $(\mathrm{ddfm}=$ satterth $)$. Os dados obtidos foram submetidos à análise de variância e regressão polinomial utilizando o PROC MIXED do SAS, versão 9.0 (SAS, 2004), adotando-se nível de significância de $5 \%$.

\section{RESULTADOS E DISCUSSÃO}

\section{CONSUMO, DIGESTIBILIDADE E DESEMPENHO PRODUTIVO}

A inclusão de grão de soja nas dietas diminui linearmente o CMS dos animais em todos os ensaios $(p<0,05$; tabelas III e IV). Esse fato se deve a menor aceitabilidade do grão de soja pelos animais, sendo que nas dietas com inclusão de altos níveis do grão, as sobras do alimento fornecido eram constituídas basicamente de grão de soja. De acordo com o NRC (2001), a adição de fontes de gordura em dietas de ruminantes normalmente resulta em diminuição do CMS. Os mecanismos pelos quais a suplementação com fontes gordura afetam o CMS não estão claros, porém podem envolver efeitos da gordura na fermentação ruminal, aceitabilidade das dietas, a liberação de hormônios intestinais, e a oxidação de gordura no fígado (Allen, 2000). Firkins and Eastridge (1994) sugeriram que o efeito hipofágico de gorduras insaturadas está ligado à redução da digestão ruminal de fibra, aumentando a

Tabela III. Consumo de matéria seca e digestibilidade aparente total da matéria seca e da FDN em função das dietas experimentais nos estudos 1 e 3 (Dry matter intake and total tract digestion of dry matter and NDF according to the

\begin{tabular}{|c|c|c|c|c|c|c|c|}
\hline & \multicolumn{4}{|c|}{ Dieta } & \multirow{2}{*}{ EPM } & \multicolumn{2}{|c|}{$p$} \\
\hline & G0 & G8 & G16 & G24 & & L & Q \\
\hline \multicolumn{8}{|c|}{ ENSAIO 1- NoVILHOS DE CORTE } \\
\hline Consumo de MS (kg/dia) & 8,88 & 8,33 & 7,95 & 8,11 & 0,22 & 0,045 & 0,225 \\
\hline \multicolumn{8}{|c|}{ Coeficiente de digestibilidade (\%) } \\
\hline Matéria seca & 66,27 & 66,62 & 64,43 & 65,59 & 0,64 & 0,446 & 0,745 \\
\hline Fibra detergente neutro & 57,06 & 53,23 & 50,17 & 52,85 & 1,13 & 0,130 & 0,160 \\
\hline Ganho médio de peso vivo (kg/dia) & 1,88 & 1,75 & 1,92 & 1,74 & 0,03 & 0,469 & 0,764 \\
\hline \multicolumn{8}{|c|}{ ENSAIO 2 - VACAS DE ALTA PRODUÇÃO } \\
\hline Consumo de MS (kg/dia) & 25,30 & 24,93 & 24,75 & 23,84 & 0,57 & 0,001 & 0,356 \\
\hline \multicolumn{8}{|c|}{ Coeficiente de digestibilidade (\%) } \\
\hline Matéria seca & 68,89 & 68,45 & 70,28 & 68,72 & 0,48 & 0,706 & 0,480 \\
\hline Fibra detergente neutro & 64,32 & 65,60 & 68,71 & 67,30 & 0,82 & 0,070 & 0,330 \\
\hline PLC (kg/dia) & 29,41 & 29,03 & 30,30 & 28,14 & 0,78 & 0,472 & 0,282 \\
\hline
\end{tabular}
experimental treatments of trials 1 and 3 ).

PLC= produção de leite corrigida para 3,5\% de gordura. G0 (Controle); G8, G16 e G24 referem-se a inclusão de 8,16 e $24 \%$ de grão de soja cru integral na dieta (com base na MS). EPM= erro padrão da média; $p=$ Probabilidades de resposta linear (L), ou quadrática $(\mathrm{Q})$. 
Tabela IV. Consumo de matéria seca e digestibilidade aparente total da matéria seca e da FDN em função das dietas experimentais no estudo 2 (Dry matter intake and total tract digestibility of dry matter and NDF according to experimental treatments in trial 2).

\begin{tabular}{|c|c|c|c|c|c|c|c|}
\hline & \multicolumn{4}{|c|}{ Dieta } & \multirow{2}{*}{ EPM } & \multicolumn{2}{|c|}{$p$} \\
\hline & G0 & G9 & G18 & G27 & & L & Q \\
\hline \multicolumn{8}{|c|}{ ENSAIO 3 - VACAS DE MÉDIA PRODUÇÃO } \\
\hline CMS kg/dia & 18,97 & 18,58 & 19,06 & 17,97 & 0,25 & 0,048 & 0,203 \\
\hline \multicolumn{8}{|c|}{ Coeficiente de Digestibilidade (\%) } \\
\hline MS & 61,80 & 62,68 & 62,01 & 62,01 & 0,72 & 0,992 & 0,725 \\
\hline FDN & 52,15 & 49,66 & 54,17 & 53,95 & 0,84 & 0,092 & 0,382 \\
\hline PLC kg/dia & 23,43 & 25,09 & 25,51 & 24,74 & 0,85 & 0,417 & 0,235 \\
\hline
\end{tabular}

$\mathrm{CMS}=$ consumo de matéria seca; $\mathrm{MS}=$ matéria seca; FDN= fibra em detergente neutro; $\mathrm{PLC}=$ produção de leite corrigida. $\mathrm{G} 0$ (Controle); G8, G16 e G24 referem-se a inclusão de 8,16 e $24 \%$ de grão de soja cru integral na dieta (com base na MS). EPM= erro padrão da média; $p=$ Probabilidades de resposta linear $(L)$, ou quadrática $(Q)$.

distensão do rúmen-retículo, causando a sensação de saciedade.

No entanto, as dietas não afetaram ( $\mathrm{p}>0,05$; tabelas III e IV) a digestibilidade da MS e da FDN. Quando fontes de gordura são fornecidas na forma de sementes oleaginosas ocorre uma lenta liberação dos lipídios no ambiente ruminal, fazendo com que a capacidade de biohidrogenção dos microorganismos não seja superada e impedindo, dessa forma, prejuízos na digestibilidade de fibra pelo efeito negativo que as gorduras insaturadas prontamente disponíveis no rúmen podem causar as bactérias fibrolíticas (Coppock \& Wilks, 1999; Palmquist, 1991).

Alguns autores verificaram queda da digestibilidade da MS devido à inclusão de fontes lipídicas (Zinn and Shen, 1996; Zinn et al., 2000; Plascencia et al., 2003) mas, em outros casos não foram encontrados efeitos da suplementação lipídica sobre a digestibilidade da dieta (Zinn, 1988; Plascencia et al., 1999). A inconsistência dos resultados está provavelmente relacionada à fonte de lipídios, a quantidade suplementada, o tipo e quantidade do volumoso utilizado (Plascencia et al., 1999). Alguns trabalhos com bovinos de corte encontraram que a suplementação de ácidos graxos em até 9,4\% da MS da dieta não afeta a digestibilidade dos nutrientes (Kucuk et al., 2004; Atkinson et al., 2006). Porém, em vacas leiteiras recomenda-se que a inclusão de gordura na dieta não ultrapasse os 6\% de EE (base na MS da dieta), mas esse limite pode ser ultrapassado se forem utilizadas fontes de gordura naturalmente protegidas (sementes oleaginosas) ou industrialmente (sais de cálcio de ácidos graxos; NRC, 2001).

O desempenho produtivo dos animais, GMD para os bovinos em terminação e a PLC para as vacas leiteiras, são características de grande importância na pro-

Tabela V. Consumo e excreção de grão de soja e composição bromatológica do grão de soja nas fezes em função das dietas experimentais de bovinos de corte (estudo 1) (Intake and fecal excretion of soybeans and chemical composition of soybeans particles on feces according to experimental treatments of trial 1).

\begin{tabular}{|c|c|c|c|c|c|c|c|}
\hline & \multicolumn{4}{|c|}{ Dieta } & \multirow{2}{*}{ EPM } & \multicolumn{2}{|c|}{$p$} \\
\hline & G0 & G8 & G16 & G24 & & $\mathrm{L}$ & Q \\
\hline \multicolumn{8}{|c|}{ Consumo } \\
\hline MS (kg/dia) & - & 0,67 & 1,27 & 1,94 & 0,13 & $<0,001$ & 0,325 \\
\hline MN (kg/dia) & - & 0,74 & 1,41 & 2,16 & 0,15 & $<0,001$ & 0,345 \\
\hline \multicolumn{8}{|c|}{ Excreção } \\
\hline $\mathrm{MN}(\%)$ & - & 0,82 & 0,96 & 1,61 & 0,15 & 0,004 & 0,129 \\
\hline MS (\%) & - & 1,90 & 2,23 & 3,50 & 0,34 & 0,003 & 0,181 \\
\hline MN (g/dia) & - & 128 & 164 & 251 & 0,03 & 0,025 & 0,568 \\
\hline MS (g/dia) & - & 52 & 70 & 100 & 0,01 & 0,021 & 0,697 \\
\hline MN corrigida & - & 56 & 79 & 109 & 0,01 & 0,022 & 0,840 \\
\hline \multicolumn{8}{|c|}{ Composição químico-bromatológica (\%) } \\
\hline MS & - & 40,33 & 42,11 & 39,26 & 0,84 & 0,595 & 0,189 \\
\hline PB & - & 41,00 & 43,29 & 45,06 & 0,97 & 0,066 & 0,886 \\
\hline EE & - & 5,82 & 5,87 & 6,00 & 0,21 & 0,665 & 0,922 \\
\hline
\end{tabular}

$\mathrm{MS}=$ matéria seca; $\mathrm{MN}=$ matéria natural; $\mathrm{PB}=$ proteína bruta; $\mathrm{EE}=$ extrato etéreo. $\mathrm{G} 0$ (Controle); $\mathrm{G} 8, \mathrm{G} 16$ e $\mathrm{G} 24$ referem-se a inclusão de 8,16 e $24 \%$ de grão de soja cru integral na dieta (com base na MS). EPM= erro padrão da média; $p=$ Probabilidades de resposta linear (L), ou quadrática $(Q)$. 
Tabela VI. Consumo e excreção de grão de soja e composicão bromatológica do grão de soja nas fezes em função das dietas experimentais de vacas de alta produção de leite (estudo 2) (Intake and fecal excretion of soybeans and chemical composition of soybeans particles on feces according to the experimental treatments of trial 2).

\begin{tabular}{|c|c|c|c|c|c|c|c|}
\hline & \multicolumn{4}{|c|}{ Dieta } & \multirow{2}{*}{ EPM } & \multicolumn{2}{|c|}{$p$} \\
\hline & G0 & G8 & G16 & G24 & & L & $Q$ \\
\hline \multicolumn{8}{|c|}{ Consumo } \\
\hline MS (Kg/dia) & - & 2,14 & 3,68 & 5,69 & 0,27 & $<0,001$ & 0,335 \\
\hline MN (Kg/dia) & - & 2,44 & 4,18 & 6,50 & 0,27 & $<0,001$ & 0,335 \\
\hline \multicolumn{8}{|c|}{ Excreção } \\
\hline $\mathrm{MN}(\%)$ & - & 3,54 & 5,76 & 7,02 & 0,36 & $<0,001$ & 0,378 \\
\hline MS (\%) & - & 7,84 & 12,55 & 14,05 & 0,80 & 0,004 & 0,181 \\
\hline MN (Kg/dia) & - & 1,70 & 2,38 & 2,74 & 0,18 & 0,002 & 0,534 \\
\hline MS (Kg/dia) & - & 0,63 & 0,91 & 1,03 & 0,06 & 0,002 & 0,453 \\
\hline MN (Kg/dia) & - & 0,69 & 0,99 & 1,13 & 0,07 & 0,001 & 0,416 \\
\hline \multicolumn{8}{|c|}{ Composição bromatológica (\%) } \\
\hline MS $(\% \mathrm{MN})$ & - & 37,22 & 38,04 & 38,03 & 0,37 & 0,337 & 0,547 \\
\hline MO & - & 96,74 & 96,74 & 96,57 & 0,07 & 0,159 & 0,377 \\
\hline PB & - & 43,65 & 43,86 & 44,53 & 0,21 & 0,066 & 0,557 \\
\hline EE & - & 9,25 & 10,02 & 10,10 & 0,32 & 0,112 & 0,434 \\
\hline FDN & - & 31,56 & 30,99 & 31,00 & 0,75 & 0,662 & 0,796 \\
\hline CNF & - & 12,24 & 11,78 & 10,87 & 0,75 & 0,362 & 0,859 \\
\hline
\end{tabular}

$\mathrm{MS}=$ matéria seca; $\mathrm{MN}=$ matéria natural; $\mathrm{PB}=$ proteína bruta; $\mathrm{EE}=$ extrato etéreo; $\mathrm{FDN}=$ fibra em detergente neutro; $\mathrm{CNF}=$ carboidrato não fibroso. G0 (Controle), G9, G18 e G27 referem-se a inclusão de 9, 18 e 27\% de grão de soja cru integral na dieta (com base na MS). $E P M=$ erro padrão da média. $p=$ Probabilidades de resposta linear $(L)$, ou quadrática $(Q)$.

dução, uma vez que estão relacionados a rentabilidade do sistema. É importante observar que apesar da queda no CMS conforme o aumento do grão de soja na dieta, o GMD dos animais em terminação e a PLC das vacas de média e de alta produção não foram prejudicados pelas dietas experimentais ( $p>0,05$, tabelas III e IV), ou seja, mesmo com menor ingestão de alimentos, os animais alimentados com alta inclusão do grão de soja mantiveram os níveis de produção. Este fato pode estar relacionado com uma maior eficiência alimentar nos animais recebendo o grão na dieta, uma vez que a suplementação lipídica melhora a eficiência da conversão de alimentos, pois ocorre uma economia no anabolismo, pois ácidos graxos pré-formados dispensam síntese de novo a partir do acetato, o que evita parte do incremento calórico associado a esta rota metabólica, pois a produção de ácidos graxos a partir de acetato demanda uma molécula de NADPH a partir do ciclo das pentoses (Sousa et al., 2009).

\section{GRÃO DE SOJA NAS FEZES}

Nas tabelas V, VI e VII estão apresentados o consumo, excreção de grão de soja e a composição bromatológica das partículas de grão de soja nas fezes. Observa-se que para os três estudos houve efeito linear crescente $(p<0,01)$ para o consumo e excreção (MS e matéria natural) do grão de soja. Ou seja, quanto maior a inclusão do grão na dieta maior será a excreção de partículas do grão nas fezes.

As dietas experimentais não afetaram a composição bromatológica das partículas de grão de soja nas fezes ( $p>0,05$, tabela V). Portanto, apesar do aumento da quantidade das partículas do grão de soja excretada, sua composição não foi alterada, devido ao coeficiente de digestibilidade não ser prejudicado pela inclusão do grão de soja nas dietas. Esses resultados indicam que mesmo a matriz lipídica sendo protegida em sementes de oleaginosas in natura, houve um aproveitamento dos lipídios contidos no grão, já que o valor de extrato etéreo do grão de soja in natura é de aproximadamente $20 \%$ e o extrato etéreo do grão excretado foi de 5,9\%. Pode-se observar, portanto, aproveitamento dos lipídios sem efeitos negativos no ambiente ruminal dos animais.

No estudo com vacas de alta produção de leite, a composição bromatológica do grão de soja presente nas fezes também não diferiu ( $p>0,05)$ para MS, MO, $\mathrm{PB}, \mathrm{EE}, \mathrm{FDNe} \mathrm{CNF}$ em função dos tratamentos (tabela VI). Destaca-se o teor de EE das partículas de grão de soja presentes nas fezes, já que o grão in natura apresenta valores de 19 a $22 \%$ de extrato etéreo, e foi verificado que as partículas do grão de soja presentes nas fezes apresentam valores médios de EE de 9,74\%, o que indica o potencial de utilização de ácidos graxos do grão de soja. De forma semelhante, no estudo com vacas de leite de média produção, também não foi o observado efeito $(p>0,05)$ para a composição bromatológica da partículas de grão de soja presente nas fezes para MS, MO, PB, EE, FDN e CNF (tabela VII). Entretanto, a composição bromatológica do grão de soja das fezes não apresentou diferença do grão in natura, mas a quantidade de grão excretada foi de $10,84 \%$ sendo o consumo de 4,68 kg de MS/dia, mostrando grande aproveitamento do grão de soja pelos animais. 
Tabela VII. Consumo e excreção de grão de soja e composição bromatológica do grão de soja nas fezes em função das dietas experimentais de vacas de média produção de leite (estudo 3) (Intake and fecal excretion of soybeans and chemical composition of soybeans particles on feces according to the experimental treatments of trial 3).

\begin{tabular}{|c|c|c|c|c|c|c|c|}
\hline \multirow{2}{*}{ Item } & \multicolumn{4}{|c|}{ Dieta } & \multirow{2}{*}{ EPM } & \multicolumn{2}{|c|}{$p$} \\
\hline & G0 & G9 & G18 & G27 & & $\mathrm{L}$ & Q \\
\hline \multicolumn{8}{|c|}{ Consumo } \\
\hline MS (kg/dia) & - & 1,66 & 3,42 & 4,68 & 0.22 & $<0,001$ & 0,037 \\
\hline MN (kg/dia) & - & 1,82 & 3,75 & 5,14 & 0,57 & $<0,001$ & 0,063 \\
\hline \multicolumn{8}{|c|}{ Excreção } \\
\hline $\mathrm{MN}(\%)$ & - & 2,08 & 2,90 & 3,66 & 0,21 & $<0,001$ & 0,181 \\
\hline MS(\%) & - & 7,05 & 8,18 & 10,84 & 0,80 & 0,004 & 0,181 \\
\hline MN (kg/dia) & - & 1,36 & 1,93 & 2,18 & 0,15 & $<0,001$ & 0,101 \\
\hline MS (kg/dia) & - & 0,18 & 0,28 & 0,33 & 0,02 & $<0,001$ & 0,123 \\
\hline MN cor (kg/dia) & - & 0,19 & 0,30 & 0,36 & 0,13 & $<0,001$ & 0,116 \\
\hline \multicolumn{8}{|c|}{ Composição bromatológica (\%) } \\
\hline MS (\%MN) & - & 31,27 & 31,13 & 32,04 & 0,49 & 0,337 & 0,547 \\
\hline MO & - & 96,14 & 96,16 & 96,19 & 0,04 & 0,657 & 0,812 \\
\hline PB & - & 54,82 & 54,73 & 54,86 & 0,11 & 0,528 & 0,796 \\
\hline EE & - & 24,25 & 24,62 & 24,68 & 0,14 & 0,412 & 0,612 \\
\hline FDN & - & 39,65 & 37,77 & 40,32 & 1,05 & 0,650 & 0,946 \\
\hline CNF & - & 32,23 & 33,76 & 31,18 & 1,05 & 0,510 & 0,748 \\
\hline
\end{tabular}

$\mathrm{MS}=$ matéria seca; $\mathrm{MN}=$ matéria natural; $\mathrm{PB}=$ proteína bruta; $\mathrm{EE}=$ extrato etéreo; $\mathrm{FDN}=$ fibra em detergente neutro; $\mathrm{CNF}=$ carboidrato não fibroso. G0 (Controle); G9, G18 e G27 referem-se a inclusão de 9, 18 e 27\% de grão de soja cru integral na dieta (com base na MS). $E P M=$ erro padrão da média; $p=$ probabilidades de resposta linear $(L)$, ou quadrática $(Q)$.

\section{CONCLUSÃO}

De acordo com os estudos avaliados, níveis crescentes de grão de soja podem ser utilizados na dieta de ruminantes sem que ocorra mal aproveitamento nutricional do grão de soja e prejuízos a produção do animal.

\section{BIBLIOGRAFIA}

Allen, M.S. 2000. Effects of diet on short-term regulation of feed intake by lactating dairy cattle. J Dairy Sci, 83: 1598-1630.

Association of Official Analytical Chemists (AOAC). 2000. Official Methods of Analysis. 17th ed. Arlington, VA.

Atkinson, R.L.; Scholljegerdes, E.J.; Lake, S.L.; Nayigihugu, V.; Hess, B.W. and Rule, D.C. 2006. Site and extent of digestion, duodenal flow, and intestinal disappearance of total and esterified fatty acids in sheep fed a high-concentrate diet supplemented with high-linoleate safflower oil. J Anim Sci, 84: 387-396.

Casali, A.O.; Detmann, E. Valadares Filho S.C.; Pereira, J.C.; Henrique, L.T.; Freitas, S.G. and Paulino, M.F. 2008. Influence of incubation time and particle size on indigestible compounds contents in cattle feeds and feces obtained in situ procedures. Braz J Anim Sci, 37: 335-342.

Coppock, C.E. and Wilks, D.L. 1991. Milk yield and composition supplemental fat in high-energy rations for lactating cows: effects on intake, digestion. J Anim Sci, 69: 3826-3837.

Côrtes, C.; Kazama, R.; da Silva- Kazama, D. Santos, G.T.D.; Zeoula, L.M. and Petit, H.V. 2015. Composition of flaxseed recovered from the faeces of dairy cows fed different proportions of whole flaxseed in the diet. J Anim Feed Sci Technol, 204: 9-17.

Firkins, J.L. and Eastridge, M.L. 1994. Assessment of the effects of iodine value on fatty acid digestibility, feed intake, and milk production. J Dairy Sci, 77: 2357-2366.
Hall, M.B. 1998. Making nutritional sense of nonstructural carbohydrates. Annual Florida Ruminant Nutrition Symposium, 9, Gainsville, F1. Proceedings... Florida University. Gainsville. pp. 108-121.

Kucuk, O.; Hess, B.W. and Rule, D.C. 2004. Soybean oil supplementation of a high-concentrate diet does not affect site and extent of organic matter, starch, neutral detergent fiber, or nitrogen digestion, but influences both ruminal metabolism and intestinal flow of fatty acids in limit-fed lambs. J Anim Sci, 82: 2985-2994.

Mertens, D.R.; Allen, M.; Carmany, J.; Clegg, J.; Davidowicz, A.; Drouches, M.; Frank, K.; Gambin, D.; Garkie, M.; Gildemeister, B.; Jeffress, D.; Jeon, C.S.; Jones, D.; Kaplan, D.; Kim, G.N.; Kobata, S.; Main, D.; Moua, X.; Paul, B.; Robertson, J.; Tayson, D.; Thiex, N.; Williams, J. and Wolf, J.M. 2002. Gravimetric determination of amylase-treated neutral detergent fiber in feeds with refluxing in beakers or crucibles: collaborative study. J AOAC Int, 85: 1217-1240.

McDonald, P.; Edwards, R.A.; Greenhalgh, J.F.D.; Morgan, C.A. 1995. Animal nutrition. $5^{\text {th }}$ ed. Addison Wesley Longman Ltd. Edinburgh Gate. Harlow. Essex. UK.

Millen, D.D. 2009. A snapshot of management practives and nutritional recommendations used by feedlot nutritionists in Brazil. J Anim Sci, 87: 3427-3439.

National Research Council - NRC. 2001. Nutrient requirements of dairy cattle. $7^{\text {th }}$ ed. rev. National Academic Press. Washington, DC.

Nocek, J.E. 1988. In situ and other methods to estimate ruminal protein and energy digestibility. A review. J Dairy Sci, 71: 2051-2069.

Palmquist, D.L. 1991. Influence of source and amount of dietary fat on digestibility in lactating cows. J Dairy Sci, 74: 1354-1360.

Paulino, M.F.; Detmann, E.; Valadares Filho, S.C. and Lana, R.P. 2002. Soja grão e caroço de algodão em suplementos múltiplos pra terminação de bovinos mestiços em pastejo. Rev Bras Zootecn, 31: 484-491.

Plascencia, A.; Estrada, M. and Zinn, R.A. 1999. Influence of free fatty acid content on the feeding value of yellow grease in finishing diets for feedlot cattle. J Anim Sci, 77: 2603-2609.

Plascencia, A.; Mendoza, G.D.; Vásquez, C. and Zinn, R.A. 2003. Relationship between body weight and level of fat supplementation on fatty acid digestion in feedlot cattle. J Anim Sci, 81: 2653-2659. 
Sklan, D.; Ashkennazi, R.; Braun, A. and Tabori, K. 1992. Fatty acids, calcium soaps of fatty acids, and cottonseeds fed to high yielding cows. J Dairy Sci, 75: 2463-2472.

Sousa, D.P.; Campos, J.M.S.; Valadares Filho, S.C.; Lana, R.P.; Sediyama, C.A.Z. and Neto, J.M. 2009. Comportamento ingestivo, consumo e digestibilidade de nutrientes, produção e composição do leite de vacas alimentadas com silagem de milho ou cana-de-açúcar com caroço de algodão. Rev Bras Zootecn, 38: 2053-2062.

Van Soest, P.J. and Mason, V.C. 1991. The influence of Maillard reaction upon the nutritive value of fibrous feeds. Anim Sci Tech, 32: 45-53.
Zinn, R.A. 1988. Comparative feeding value of supplemental fat in finishing diets for feedlot steers with and without monensin. J Anim Sci, 66: 213-227.

Zinn, R.A. and Shen, Y. 1996. Interaction of dietary calcium and supplemental fat on digestive function and growth performance in feedlot steers. J Anim Sci, 74: 2303-2309.

Zinn, R.A.; Gulati, S.K.; Plascencia, A. and Salinas, J. 2000. Influence of ruminal biohydrogenation on the feeding value of fat in finishing diets for feedlot cattle. J Anim Sci, 78: 1738-1746. 